(c) 2021 Universidad Nacional Autónoma de México, Facultad de Estudios Superiores Zaragoza.

Este es un artículo Open Access bajo la licencia CC BY-NC-ND (http://creativecommons.org/licenses/by-nc-nd/4.0/).

TIP Revista Especializada en Ciencias Químico-Biológicas, 24: 1-12, 2021.

https://doi.org/10.22201/fesz.23958723e.2021.369

\title{
Efecto de la fotolisis y pH en la degradación de la oxitetraciclina en sedimento y agua marina
}

\author{
Karen Griselda Sotelo-Cornejo ${ }^{1}$, Angelica Espinosa-Plascencia ${ }^{1}$, \\ Sergio Gámez-Bayardo ${ }^{2}$, Adolfo Pérez-Álvarez, Maribel Jiménez-Edeza², \\ Alfonso García-Galaz ${ }^{1 a}$ y María del Carmen Bermúdez-Almada ${ }^{1 *}$ \\ ${ }^{1}$ Laboratorio de Análisis Biológicos; ${ }^{1 a}$ Laboratorio de Microbiología Polifásica y Bioactividades, \\ Coordinación de Ciencia de los Alimentos, Centro de Investigación en Alimentación y Desarrollo, A.C. \\ Carretera Gustavo Enrique Astiazarán Rosas \# 46. La Victoria, Hermosillo 83304, Sonora, México. \\ ${ }^{2}$ Laboratorio de Investigación y Diagnóstico Microbiológico, Facultad de Ciencias Químico-Biológicas, \\ Universidad Autónoma de Sinaloa. Culiacán 80013, Sinaloa, México. E-mail: *cbermudez@ciad.mx
}

\section{RESUMEN}

La oxitetraciclina (OTC) es un antibiótico frecuentemente utilizado en el control de enfermedades provocadas por Vibrio en granjas de cultivo de camarón Penaeus vannamei. Se evaluó la degradación de la OTC en muestras de sedimento y agua marina $(2: 98 \mathrm{p} / \mathrm{v})$, con OTC como estándar y OTC adicionada en alimento para camarón, en ambos casos a 10 $\mu \mathrm{gmL}^{-1}$. Los ensayos tuvieron una duración de 40 días, y se evaluaron factores abióticos como exposición a la luz y efecto del pH (7 y 8). Las muestras con estándar de OTC expuestas a la luz, tuvieron una degradación del antibiótico del $100 \%$ para ambos valores de $\mathrm{pH}$; en las muestras no expuestas, la degradación fue de $88.33 \%$ a pH 7 y $90.90 \%$ a pH 8. En las muestras con OTC en alimento para camarón, la degradación fue del $100 \%$ a los 40 días, con y sin exposición a la luz y con los dos valores de $\mathrm{pH}$. El tiempo de vida media de degradación de la OTC en el alimento, fue menor con respecto a las muestras adicionadas con estándar con y sin exposición a la luz $(P \leq 0.05)$. Los residuos de la OTC se degradan por efecto de la luz y $\mathrm{pH}$, reduciendo su permanencia en los sedimentos acuícolas.

Palabras clave: oxitetraciclina, sedimento y agua marina, alimento para camarón, resistencia bacteriana.

\section{Effect of photolysis and pH on the degradation of oxytetracycline in sediment and seawater}

\begin{abstract}
Oxytetracycline (OTC) is an antibiotic frequently used in the control of diseases caused by Vibrio in Penaeus vannamei shrimp farms. The degradation of OTC was evaluated in samples of sediment and seawater $(2: 98 \mathrm{w} / \mathrm{v})$, one with an OTC standard and another with OTC in shrimp feed, both at $10 \mu \mathrm{gmL}^{-1}$. The tests lasted 40 days, and the abiotic factors were evaluated, such as the exposure to light, and the effect of the $\mathrm{pH}$ (7 and 8). The samples with OTC standard exposed to light, had a degradation of the antibiotic of $100 \%$ for both $\mathrm{pH}$ values; In the unexposed samples to light, the degradation was $88.33 \%$ at $\mathrm{pH} 7$ and $90.90 \%$ at $\mathrm{pH} 8$. In the samples with OTC in shrimp feed, the degradation was $100 \%$ at 40 days, with and without exposure to light, at different $\mathrm{pH}$ values. The half-life time of OTC degradation in shrimp feed was lower with due to the samples added with standard with and without exposure to light $(P \leq 0.05)$. OTC residues can be degraded by the effect of light and $\mathrm{pH}$, reducing their permanence in aquaculture sediments.

Keywords: oxytetracycline, sediment and seawater, shrimp feed, bacterial resistance.
\end{abstract}




\section{INTRODUCCIÓN}

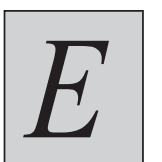

1 consumo y producción de alimentos provenientes del mar se ha incrementado debido al crecimiento de la población mundial en los últimos años. Sin embargo, el sector acuícola no está exento de la aparición de enfermedades durante la producción de alimentos de origen marino, con pérdidas económicas importantes (FAO, 2018). Una de las alternativas para el control de las enfermedades en el cultivo de peces y crustáceos ha sido el uso de antibióticos. Su aplicación con fines profilácticos ha disminuido en algunos países, mientras que en otros, como Asia, se siguen administrando antibióticos en un $80 \%$ a las especies marinas cultivadas (Institute, 2016). En China, se han empleado 13 distintos antibióticos, comparado con el Reino Unido que ha utilizado cinco de ellos con amplio espectro, en las actividades acuícolas (Topp, Joakim, Miller, Van den Eede \& Virta, 2018). En México, los antibióticos como la oxitetraciclina (OTC), enrofloxacina y florfenicol se usan en la industria camaronícola para controlar las infecciones bacterianas que se presentan durante los ciclos de producción (Soto-Rodríguez, Gómez-Gil, Roque \& Lozano, 2008).

La oxitetraclicina se caracteriza por tener un amplio espectro de acción en la medicina veterinaria. Es uno de los antibióticos más utilizados en el sector acuícola por poseer actividad bactericida y ser un fármaco de bajo costo (Leal, Esteves \& Santos, 2016). Químicamente, la estructura de la OTC la forman anillos poliaromáticos que le confieren estabilidad y una vida media $\left(\mathrm{t}^{1} / 2\right)$ relativamente larga en el medio ambiente, incrementando su permanencia como contaminante (Yan et al., 2018). El mecanismo de acción de la OTC es por inhibición de la síntesis de proteínas mediante el bloqueo de la función de la subunidad 30S del ribosoma, impidiendo el crecimiento bacteriano(GómezGil, Roque \& Guerra, 2014). Cabe mencionar que la OTC no se metaboliza completamente durante su administración terapéutica, excretándose de forma inalterada al medio ambiente y en el agua, siendo las características fisicoquímicas del fármaco y del medio receptor importantes en su distribución, transporte y acumulación en el agua y en sedimento (Prado, Romalde \& Barja, 2010; Acevedo, Severiche \& Jaimes, 2015).

La fotodegradación es una de las principales formas de degradación de la OTC en aguas superficiales, sin embargo es también afectada por la formación de complejos con iones metálicos como el $\mathrm{Ca}^{2+}$ y el $\mathrm{Mg}^{2+}$ (Andreozzi, Raffaele \& Nicklas, 2003). La presencia de materia orgánica disuelta y distintos iones en los ambientes acuáticos influyen en el comportamiento de la fotolisis en los antibióticos (Jiao, Meng, Yin, Wang \& Chen, 2008). La OTC posee poca estabilidad en condiciones ambientales abióticas como son el $\mathrm{pH}$ y la exposición a la luz (Rojas, 2010), siendo la fotolisis el principal mecanismo de su degradación (Oka et al., 1989; Doi \& Stoskopf, 2000; Hong-Thih \& Jing-Ju, 2009). El pH tiene un papel importante en la formación de complejos con distintos iones presentes en el agua marina. El catión de la OTC es considerado un ácido triprótico, presentando actividad de disociación ácida $\left(\mathrm{pK}_{\mathrm{a}}\right)$ de 3.2-3.6, 7.3-7.5 y 8.9-9.9 (Kulshrestha, Giese \& Aga, 2004; Jiao et al., 2008). Estudios previos han comprobado que la degradación de la OTC en el agua marina es más rápida por fotolisis, mientras que en los sedimentos se relaciona con la actividad microbiana (Samuelsen, 1989; Pouliquen, Le Bris \& Pinault, 1992; Pouliquen, Delépée, Larhantec-Verdier, Morvan \& Le, 2007; Hong-Thih \& Jing-Ju, 2009; Leal et al., 2016; González-Gaya, Cherta, Nozal \& Rico, 2018). El uso de la luz solar como factor de degradación de la OTC ha sido una alternativa efectiva y de bajo costo. Sin embargo, la cinética de degradación puede ser dependiente de la composición iónica del agua marina y de los compuestos orgánicos presentes (Leal et al., 2016).

Una de las principales causas de residuos de OTC acumulados en los sedimentos de los estanques acuícolas es el alimento adicionado con este antibiótico que no es ingerido por los organismos, y corresponde a un aproximado entre $70-80 \%$. Otra fuente de contaminación son las heces de los camarones, que contienen OTC sin metabolizar y se lixivia en los sistemas de cultivo (Li \& Zhang, 2016; Sosa, Medina \& Faure, 2013; Kong et al., 2012; Oka, et al., 1985). Los residuos de OTC y sus metabolitos epímeros se han detectado en el suelo, agua y en productos alimenticios, en concentraciones que van desde nanogramos (ppb), hasta microgramos (ppm) (Yan et al., 2018). Además, se ha demostrado que la OTC es el antibiótico mayormente detectado en los productos importados provenientes de la acuicultura de diversos países (Topp et al., 2018).

La permanencia de los residuos de antibióticos en los sistemas de cultivo acuícola, incrementa la resistencia antimicrobiana en las bacterias patógenas, lo que ocasiona pérdidas cuantiosas en la industria camaronícola. Por ello, el objetivo de esta investigación fue determinar el efecto de la fotolisis y el $\mathrm{pH}$ en la degradación de la oxitetraciclina en solución y contenida en el alimento para camarón, en muestras de sedimento y agua marina, para conocer la residualidad del antibiótico en estanques de cultivo de camarón $P$. vannamei.

\section{Materiales Y MÉTOdoS}

\section{Obtención y preparación de las muestras}

Se realizó la toma de muestras de sedimento y agua de mar en una granja de cultivo de camarón $P$. vannamei ubicada en Bahía de Kino, Sonora (2841'54.5’N 11149’59.5’W). Se seleccionó esta granja debido a que se tuvo comunicación personal con el administrador técnico de la misma, quien nos informó que por política de la empresa desde hace 10 años no se aplican tratamientos con antibióticos como parte del proceso de cultivo del camarón.

Tanto las muestras de agua del mar como de sedimento, se tomaron de cuatro estanques de la granja seleccionados al azar, 
colectándolas en seis diferentes puntos de cada estanque. En cada muestreo se tomaron $20 \mathrm{~L}$ de agua de mar y se colocaron en recipientes de plástico debidamente identificados. En el caso del sedimento, se tomaron muestras de $1 \mathrm{~kg}$, empleando para su recolección una draga metálica introducida en el estanque a $20 \mathrm{~cm}$ de profundidad. Las muestras se colocaron en bolsas de plástico estériles adecuadamente identificadas. Todas las muestras se trasladaron en hieleras con refrigerantes, al Laboratorio de Análisis Biológicos del Centro de Investigación en Alimentación y Desarrollo, A.C. (CIAD), donde se almacenaron en condiciones de refrigeración hasta su uso.

En el laboratorio las muestras se manejaron de la siguiente manera: para el agua del mar, el volumen total recolectado en los estanques se mezcló en un solo recipiente y se homogeneizó. En el caso del sedimento, todas las muestras colectadas se vaciaron también en un recipiente y se homogeneizaron. En las muestras de sedimento se determinó la humedad con la finalidad de considerar el peso en seco para la preparación de la muestra de sedimento y agua marina, siguiendo el método descrito por Tirado, Montero \& Acevedo (2015). Además, el sedimento y el agua marina fueron esterilizados de manera independiente, a $121^{\circ} \mathrm{C} / 30 \mathrm{~min}$, para eliminar microrganismos que pudieran afectar la degradación del antibiótico, y sólo observarla por efecto del $\mathrm{pH}$ y la luz.

Se confirmó la ausencia de la OTC en las muestras de sedimento y del agua del mar, para posteriormente preparar una mezcla homogénea de ambas, en una proporción 2:98 (p/v), de la que se tomaron las submuestras para la realización de los ensayos.

\section{Análisis fisicoquímicos}

En el caso de las muestras de agua del mar se realizaron mediciones del $\mathrm{pH}$ utilizando un potenciómetro YSI (Ecosense $\mathrm{pH}$ 10, Yellow Springs, OH. EUA); salinidad con un refractómetro Aquatic Eco-Systems (VitalSine SR-6, Apopka, FL.EUA), oxígeno disuelto (OD) y temperatura con un oxímetro YSI (YSI 55, Yellow Springs OH. EUA).

\section{Alimento para camarón}

Los alimentos para camarón con y sin OTC que se utilizaron en el ensayo fueron de origen comercial. Antes de iniciar el ensayo se hizo la determinación de OTC en muestras de ambos alimentos, en la primera para conocer la concentración del antibiótico, y en la segunda para confirmar la ausencia de éste. Estos análisis se realizaron mediante cromatografía de líquidos de alta resolución.

Estandarización del método cromatográfico para la determinación de la oxitetraciclina en el sedimento y agua marina

La determinación de la OTC en el sedimento y agua marina se hizo mediante un método de cromatografía de líquidos de alta resolución (HPLC), empleando un estándar de referencia de alta pureza (Sigma-Aldrich, EUA). Se llevó a cabo la validación del método analítico evaluando los parámetros de linealidad, especificidad, límite mínimo de detección(LMD), límite mínimo de cuantificación (LMC) y exactitud. La linealidad del sistema se evaluó mediante curvas de calibración de la OTC en un intervalo de concentraciones de $0.03 \mathrm{a} 10 \mu \mathrm{gmL}^{-1}$, las diluciones se prepararon con una solución de ácido oxálico $0.02 \mathrm{M}$ y acetonitrilo grado cromatográfico, en proporción 80:20 (v/v). Para determinar la linealidad del método se emplearon muestras de sedimento y agua marina adicionadas con el antibiótico en el mismo rango de concentraciones que se usaron para la linealidad del sistema. Estos análisis se realizaron por duplicado de extracción y triplicado de inyección en el cromatógrafo de líquidos de alta resolución. La especificidad o selectividad se observó mediante la resolución del analito en el cromatograma obtenido del análisis de la muestra de sedimento y agua marina con el antibiótico. La exactitud se estableció utilizando muestras adicionadas con OTC a una concentración conocida, para obtener el porcentaje de recuperación. Los solventes empleados para la cuantificación de la OTC en las muestras de sedimento y agua marina fueron acetonitrilo y metanol grado cromatográfico (Fermont, Mex.).

El equipo utilizado para la determinación del antibiótico fue un Cromatógrafo de Líquidos de Alta Resolución (HPLC) Varian ProStar 210, un detector UV-Vis Varian ProStar 325 a una longitud de onda de $365 \mathrm{~nm}$, una columna Nucleosil $\mathrm{C}_{18}$ de $150 \times 4.6 \mathrm{~mm}$, con tamaño de partícula de $5 \mu \mathrm{m}$. La fase móvil consistió en ácido oxálico $0.02 \mathrm{M}$ y acetonitrilo grado cromatográfico en proporción 80:20 (v/v), a un flujo isocrático de $1 \mathrm{~mL} / \mathrm{min}$ y un tiempo de corrida de $13 \mathrm{~min}$. El área bajo la curva fue el parámetro que se consideró para determinar la concentración de la OTC en las muestras, de acuerdo al método de estándar externo.

\section{Ensayo de degradación de la oxitetraciclina por factores abióticos}

Para evaluar la degradación de la OTC en las muestras de sedimento y agua marina por efecto del $\mathrm{pH}$ y la exposición a la luz, se realizó un ensayo bajo condiciones controladas en el Laboratorio de Análisis Biológicos del CIAD. El ensayo se llevó a cabo por un período de 40 días, siguiendo el protocolo metodológico publicado por Lai \& Lin (2009). Se emplearon 12 frascos de vidrio de $250 \mathrm{~mL}$, a los que se añadieron $100 \mathrm{mLde}$ la muestra de sedimento y agua marina $(2: 98 \mathrm{p} / \mathrm{v})$. El pH de esta muestra se ajustó a 7 y 8 , empleando soluciones amortiguadoras de $\mathrm{HCl}$ y $\mathrm{NaOH} 0.1 \mathrm{M}$.

Para llevar a cabo el ensayo, se tuvieron dos grupos de tratamientos, Tratamiento 1: degradación del estándar de OTC (con y sin exposición a la luz, pH 7 y 8); Tratamiento 2 : degradación de la OTC contenida en alimento para camarón (con y sin exposición a la luz, pH 7 y 8). En cada tratamiento seis frascos de $250 \mathrm{~mL}$ se mantuvieron descubiertos y seis 
se cubrieron totalmente con papel aluminio para evitar la exposición de la OTC a la luz. El efecto de la luz se dio a través de una lámpara led con una intensidad lumínica de $80 \mathrm{~mW} / \mathrm{cm}^{2}$ que es cercana al valor promedio del espectro de luz visible, simulando la luz solar.

Dentro de cada uno de los tratamientos ( 1 y 2 ), se hizo la siguiente distribución: dos frascos fueron utilizados como controles negativos, uno de ellos contenía la muestra de sedimento y agua marina y el otro el alimento para camarón sin OTC. A otros dos frascos de cada tratamiento se les adicionó un estándar de OTC a una concentración teórica de $10 \mu \mathrm{gmL}^{-1}$. A los dos últimos frascos de cada tratamiento se les agregó alimento para camarón con OTC a una concentración teórica de $10 \mu \mathrm{gmL}^{-1}$. Durante el ensayo todos los frascos se mantuvieron en agitación mecánica a $250 \mathrm{rpm}$ y $25^{\circ} \mathrm{C}$ por 40 días. De cada frasco se tomaron muestras de $3 \mathrm{~mL}$ de sedimento y agua marina, en distintos lapsos de tiempo de: 0, 0.5, 1, 2, 4, 7, 9, 11, 14, 17, 20 y 40 días. Estas muestras se colocaron en viales color ámbar debidamente etiquetados y se almacenaron en un congelador TOREY a $-20{ }^{\circ} \mathrm{C}$ para el análisis de la OTC por HPLC.

El procedimiento anterior se llevó a cabo en los tratamientos 1 y 2 a pH 7 y pH 8, siempre bajo las mismas condiciones experimentales.

\section{Determinación de la oxitetraciclina en las muestras de sedimento y agua marina}

La determinación de la OTC en las muestras de sedimento y agua marina se realizó con base en el procedimiento de Pouliquen et al. (1992). Estos análisis se llevaron a cabo por duplicado de extracción y triplicado de inyección en el HPLC. Para asegurar la calidad de los resultados, en el análisis de las muestras se incluyó un control positivo que consistió en una muestra de sedimento y agua marina adicionada con OTC a una concentración de $2 \mu \mathrm{gmL}^{-1}$. Esta muestra se empleó para determinar el porcentaje de recuperación de la OTC (exactitud del método), también se incluyó el análisis de una muestra de sedimento y agua marina sin OTC como control negativo.

La extracción de la OTC del sedimento y agua marina se realizó tomando $1 \mathrm{~mL}$ de la muestra y se colocó en tubos cónicos de plástico de $15 \mathrm{~mL}$ que fueron agitados en un vortex por $30 \mathrm{~s}$ (Thermo Scientific, EUA) y sonicados por 15 min (Sonicador Cole Parmer, mod. 8892, Chicago, IL. EUA). Posteriormente, los tubos se centrifugaron a $2,580 \mathrm{~g}$ por $10 \mathrm{~min}$ a $4{ }^{\circ} \mathrm{C}$ en una centrífuga Heraeus Megafuge 8 Benchtop (Thermo Scientific, EUA). Los sobrenadantes de las muestras se filtraron utilizando una jeringa de plástico de $3 \mathrm{~mL}$ y un filtro (Acrodisc LC13 PVDF), con un tamaño de poro de $0.2 \mu \mathrm{m}$. El filtrado se colectó en viales de $2 \mathrm{~mL}$ para inyectar $200 \mu \mathrm{L}$ de muestra en el equipo de HPLC.

\section{Análisis estadístico de los datos}

Durante el desarrollo de los ensayos de degradación de la OTC, las variables abióticas como, la salinidad, $\mathrm{pH}$, oxígeno disuelto y temperatura fueron analizadas mediante estadística descriptiva (media y desviación estándar). Para la evaluación de los datos obtenidos de la determinación de humedad en el sedimento, se empleó un análisis de varianza (ANOVA) de una vía y la diferencia entre medias se obtuvo aplicando la prueba de Tukey-Kramer.

Los resultados de la concentración de la OTC en las muestras de sedimento y agua marina se analizaron utilizando un ANOVA multifactorial, incluyendo como factores la luz, el pH, la OTC (estándar de OTC y OTC en alimento), y el tiempo. Las diferencias obtenidas entre las medias de cada una de las variables en la degradación de la OTC fueron analizadas con la prueba de Bonferroni. Un valor de $P \leq 0.05$ se consideró estadísticamente significativo. El análisis estadístico de los datos se realizó empleando el programa computacional Minitab versión 19.1.0.0.

Los datos de la cinética de degradación de la oxitetraciclina fueron evaluados mediante un modelo de regresión no lineal de primer orden mediante la siguiente ecuación:

$$
\frac{\mathrm{C}}{\mathrm{C}_{0}}=\mathrm{e}^{-\mathrm{kt}}
$$

En la cual $k$ es el rango constante de primer orden, $t$ es el tiempo transcurrido, $C_{0}$ y $C$ son las concentraciones de OTC inicial y final respectivamente (Leal et al., 2016). El modelo no lineal fue ajustado mediante el software GraphPad Prism 9.0.2.

\section{Resultados \\ Parámetros fisicoquímicos en la muestra de sedimento y agua marina}

La evaluación de los parámetros fisicoquímicos como: $\mathrm{pH}$, salinidad, oxígeno disuelto y temperatura, que presentaron los microambientes dentro de los frascos con las muestras de sedimento y agua marina, mostró valores que se mantuvieron conforme a los óptimos en los sistemas de cultivo de camarón según lo reportado por Li et al. (2008) y Venkateswara-Rao (2009) (Tabla I).

Tabla I. Parámetros fisicoquímicos en muestras de sedimento y agua marina.

\begin{tabular}{|c|c|}
\hline Parámetro & Valor obtenido \\
\hline Salinidad & $37 \pm 2.37 \%$ o \\
\hline $\mathrm{pH}$ & $7.72 \pm 0.47$ \\
\hline Oxígeno disuelto & $4.3 \pm 0.45 \mathrm{mgL}^{-1}$ \\
\hline Temperatura & $28 \pm 0.50^{\circ} \mathrm{C}$ \\
\hline
\end{tabular}




\section{Alimento para camarón}

El resultado obtenido en el análisis de oxitetraciclina en el alimento comercial para camarón fue de $3256.51 \pm 38.97{\mu \mathrm{gg}^{-1}}^{-1}$ para el alimento medicado (adicionado con OTC), y se confirmó la ausencia del antibiótico en el alimento sin medicar (sin adición de OTC). Con base en la concentración obtenida de OTC en el alimento, se hicieron los cálculos para tener una concentración de $10 \mu \mathrm{gmL}^{-1}$ en las muestras de sedimento y agua marina utilizadas en los ensayos.

Estandarización del método cromatográfico para determinar oxitetraciclina y aseguramiento de la calidad de los datos Antes de utilizar para el ensayo las muestras independientes de sedimento y agua marina colectadas en la granja acuícola, se analizaron para confirmar la ausencia de la OTC mediante cromatografía de líquidos de alta resolución.

La validación del método analítico empleado para la determinación de la OTC en las muestras de sedimento y agua marina mostró buenos resultados en los parámetros obtenidos. En la linealidad del método, se obtuvo un coeficiente de determinación $\mathrm{r}^{2}=0.996$ y en la linealidad del sistema $\mathrm{r}^{2}=0.999$. Se determinó un límite de detección para OTC de $0.06 \mu \mathrm{gmL}^{-1}$ y un límite de cuantificación de $0.1 \mu \mathrm{gmL}^{-1}$. El parámetro de exactitud evaluado a través del porcentaje de recuperación de la OTC en muestras de sedimento y agua marina adicionadas con una concentración conocida del antibiótico fue de $104.0 \pm 25.5 \%$. El método implementado demostró ser simple, rápido, económico y confiable. Los resultados obtenidos en los parámetros de validación aseguran la calidad de los datos generados.

\section{Degradación del estándar de la oxitetraciclina en las muestras de sedimento y agua marina}

En las muestras de sedimento y agua marina, analíticamente la OTC mostró un tiempo de retención de $8.25 \pm 0.02 \mathrm{~min}$, observándose en el cromatograma un pico con buena resolución, sin interferencia de otros compuestos, lo que nos indica una adecuada especificidad o selectividad del método. La Figura 1 presenta cromatogramas típicos obtenidos de muestras de sedimento y agua marina con OTC y un estándar de referencia de la OTC a una concentración de $10 \mu \mathrm{gmL}^{-1}$.

Los resultados del experimento de degradación de la OTC sin exposición a la luz y a pH 7 y 8 , mostraron que para el estándar de OTC contenido en las muestras de sedimento y agua marina, al día 20 del ensayo, en los frascos protegidos de la luz a pH 7, se tuvo una degradación del antibiótico de $75.16 \%$ y para el $\mathrm{pH}$ 8 fue de $69.65 \%$. Hubo un incremento en la degradación de la OTC con el tiempo, detectándose a los 40 días del ensayo una concentración del antibiótico de $0.86 \pm 0.04 \mu \mathrm{gmL}^{-1}$, lo que representó una degradación del $88.33 \%$ con respecto al valor inicial $\left(7.37 \pm 0.46 \mu \mathrm{gmL}^{-1}\right)$ en los frascos con $\mathrm{pH} 7$; y una concentración de $0.71 \pm 0.23 \mu \mathrm{gmL}^{-1}$ en los frascos con $\mathrm{pH} 8$, donde la concentración inicial fue de $7.81 \pm 0.14 \mu \mathrm{gmL}^{-1}$, lo que indica una degradación del 90.9\% (Figura 2). Para el caso de las muestras de sedimento y agua marina con estándar de OTC a pH 7 y 8 , y expuestas a la luz, no se detectó el antibiótico al finalizar el ensayo (40 días), para ambos valores de $\mathrm{pH}$.

El análisis estadístico demostró que el efecto del pH en la degradación de la OTC en los sistemas con y sin exposición a la luz, y entre los distintos puntos de muestreo, no presentó diferencias significativas $(P \geq 0.05)$. Sin embargo, la interacción de los factores como la luz, el tipo de OTC y el tiempo de evaluación, mostraron su efecto sobre la degradación del antibiótico (Tabla II). La prueba estadística de Bonferroni demostró que existe diferencia significativa en la degradación de la OTC conforme al tiempo transcurrido y los diversos factores evaluados $(P \leq 0.05)$.

La aplicación del método de regresión no lineal y su validación a través del coeficiente de determinación $\left(\mathrm{r}^{2}\right)$ no fue suficiente para establecerlo como método de predicción de degradación del antibiótico, debido a que el comportamiento de los datos es discontinuo. Es por ello, que evaluar el comportamiento del error estándar se consideró indispensable como método confirmatorio de precisión del modelo matemático utilizado (Tabla III).

Los resultados obtenidos en las muestras de sedimento y agua marina adicionadas con el estándar de OTC, mantenidas a pH 7 y con exposición a la luz, mostraron un tiempo de vida media de degradación ( $\mathrm{t}$ 1/2) de $44.37 \pm 21.08 \mathrm{~min}$, a diferencia de las muestras no expuestas a la luz donde el $t \frac{1}{2}$ fue de $117.44 \pm 77.26 \mathrm{~min}$. En los frascos mantenidos a $\mathrm{pH} 8$ y expuestos a la luz, el t $1 / 2$ de degradación de la OTC fue de $49.68 \pm 15.0 \mathrm{~min}$, y en los que se mantuvieron sin exposición a la luz, el t $1 \frac{1}{2}$ se prolongó hasta $63.85 \pm 18.98 \mathrm{~min}$ del ensayo, requiriéndose más tiempo para la degradación del antibiótico en el microambiente (Tabla III).

\section{Degradación de la oxitetraciclina en alimento para camarón en las muestras de sedimento y agua marina}

En los frascos con sedimento y agua marina expuestos a la luz y a pH 7, la concentración inicial de la OTC proveniente del alimento para camarón fue de $3.0 \pm 1.27 \mu \mathrm{gmL}^{-1}$, y a las $12 \mathrm{~h}$ de iniciado el ensayo se incrementó a $6.4 \pm 0.42 \mu \mathrm{gmL}^{-1}$, mientras que en los frascos no expuestos a la luz la concentración del antibiótico se incrementó de $2.05 \pm 0.07$ a $7.30 \pm 0.84 \mu \mathrm{gmL}^{-1}$ a las $24 \mathrm{~h}$. Las concentraciones de la OTC obtenidas a las 12 y $24 \mathrm{~h}$ fueron las que se tomaron en cuenta para determinar el porcentaje de degradación del antibiótico. La degradación de la OTC en el alimento para camarón de la muestra de sedimento y agua marina, se presenta en la Figura 3. Trascurridos 20 días del ensayo, la OTC se detectó solamente en los frascos con las muestras de sedimento y agua marina protegidos de la luz, en niveles de $0.29 \pm 0.007 \mu \mathrm{gmL}^{-1}$, lo que representó una 

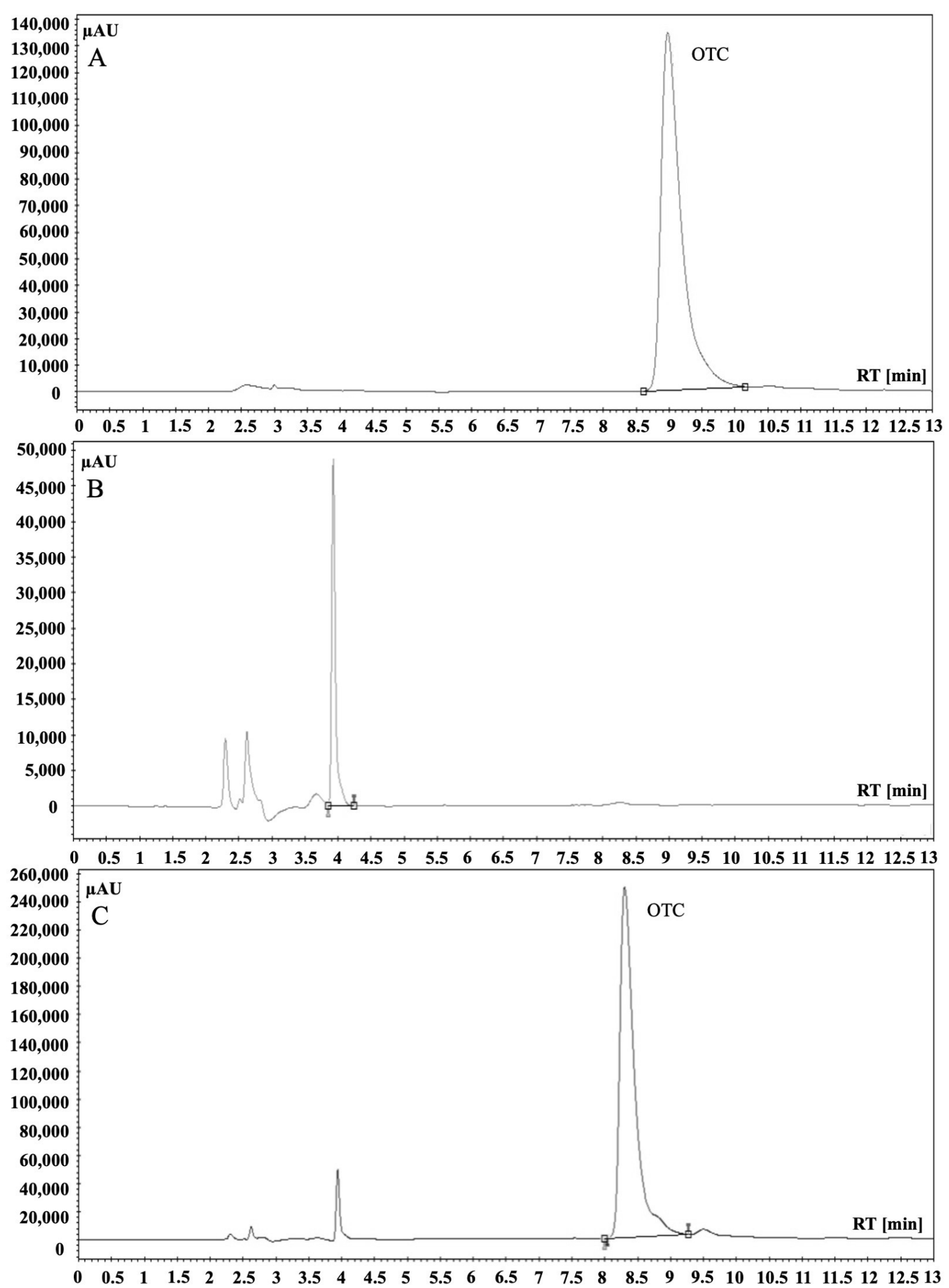

Figura 1. Cromatogramas de: A) Estándar de OTC a $10 \mu \mathrm{gmL}^{-1}$; B) Muestra de sedimento y agua marina sin OTC; C) Muestra de sedimento y agua marina con estándar de OTC a $10 \mu \mathrm{gmL}^{-1}$. 
A)

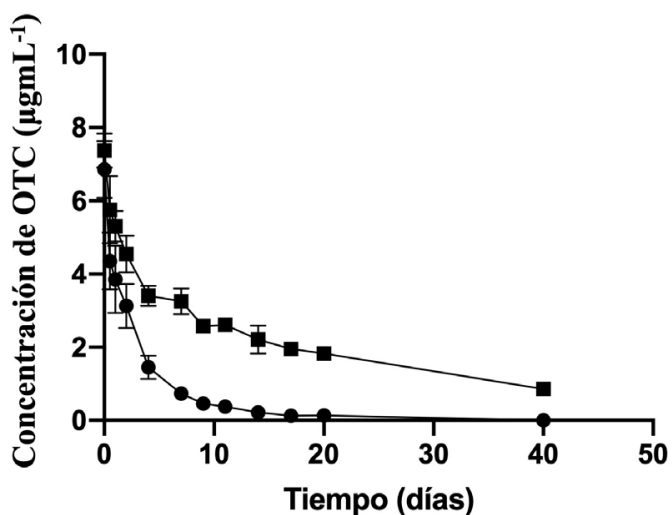

- Sedimento y agua marina expuestos a la luz

- Sedimento y agua marina sin exposición a la luz
B)

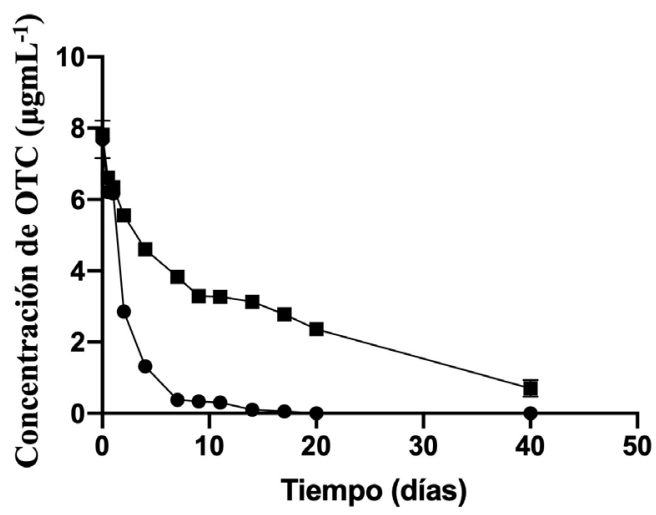

- Sedimento y agua marina expuestos a la luz

- Sedimento y agua marina sin exposición a la luz

Figura 2. Cinética de degradación de la OTC como estándar por efecto de la luz y pH en muestras de sedimento y agua marina. A) Degradación de la OTC a pH 7. B) Degradación de la OTC a pH 8.

Tabla II. Análisis de varianza multifactorial de los datos de degradación y cuantificación de la OTC en las muestras de sedimento y agua marina.

\begin{tabular}{|c|c|c|c|c|c|}
\hline Variable & $\begin{array}{c}\text { Grados de } \\
\text { Libertad }\end{array}$ & $\begin{array}{c}\text { Suma de Cuadrados } \\
\text { Ajustada }\end{array}$ & $\begin{array}{c}\text { Media de Cuadrados } \\
\text { Ajustada }\end{array}$ & Valor de F & Valor de P \\
\hline $\mathrm{pH}$ & 1 & 2.75 & 2.7528 & 2.55 & 0.112 \\
\hline Luz & 1 & 63.61 & 63.6066 & 18.4574 & 17.07 \\
\hline Tipo de OTC & 1 & 18.46 & 87.5475 & 80.99 & $0.000^{*}$ \\
\hline Tiempo & 11 & 963.02 & 1.0810 & 11.91 \\
\hline Error & 177 & 191.33 & 2.1483 & $0.000^{*}$ \\
\hline Ajuste & 81 & 174.01 & 0.1804 & & \\
\hline Error puro & 96 & 17.32 & 1239.17 & & \\
\hline Total & 191 & & & \\
\hline
\end{tabular}

*: Valor de $P \leq 0.05$ estadísticamente significativo.

degradación del $97.16 \%$ del antibiótico respecto a la más alta concentración de OTC, alcanzada a las 12 h. Esta concentración residual de la OTC se encuentra por arriba del Límite Máximo Permitido $\left(0.1 \mu \mathrm{gmL}^{-1}\right)$ tomado como referencia, debido a que no existe un LMP para este tipo de muestras. Al finalizar el ensayo (40 días) no se detectó la OTC en los frascos que contenían la muestra de sedimento y agua marina y alimento para camarón con el antibiótico (Figura 3).

En los frascos con sedimento y agua marina a $\mathrm{pH} 8$ expuestos a la luz, inicialmente se detectó una concentración de $5.43 \pm 2.42 \mu \mathrm{gmL}^{-1}$ de OTC, esta concentración se incrementó a $7.25 \pm 0.01 \mu \mathrm{gmL}^{-1}$ a las $12 \mathrm{~h}$; y en los frascos que no se expusieron a la luz el incremento en la concentración de OTC fue de $3.77 \pm 0.26$ a $7.60 \pm 0.05 \mu \mathrm{gmL}^{-1}$ a las $12 \mathrm{~h}$. A los 20 días del ensayo en los frascos con sedimento no expuestos a la luz, hubo una concentración de $0.22 \pm 0.01 \mu \mathrm{gmL}^{-1}$ de OTC (nivel superior al LMP), lo que significa una degradación del antibiótico del $97.80 \%$, respecto a la concentración alcanzada a las $12 \mathrm{~h}$ de iniciado el experimento. En los frascos que fueron expuestos a la luz no se detectó el antibiótico al final del experimento (40 días) (Figura 3). De acuerdo con el análisis de varianza multifactorial, la interacción de los factores mostró un efecto significativo $(P \leq 0.05)$, a partir de las $12 \mathrm{~h}$ de iniciado el ensayo de degradación de la OTC para ambos tratamientos (con y sin exposición a la luz). 
Tabla III. Cinética de degradación in vitro de la oxitetraciclina en sedimento y agua marina.

\begin{tabular}{|c|c|c|c|c|c|c|c|}
\hline & Muestras & $\mathbf{n}$ & pH & $k\left(\min ^{-1}\right) \pm D E$ & t $1 / 2\left(\min ^{-1}\right) \pm D E$ & $\mathbf{r}^{2}$ & $\begin{array}{c}\text { Error } \\
\text { estándar }\end{array}$ \\
\hline & $\begin{array}{l}\text { estándar de OTC } \\
\text { expuesto a la luz }\end{array}$ & 12 & & $0.022 \pm 0.005$ & $44.37 \pm 21.08$ & 0.9895 & 0.2025 \\
\hline & $\begin{array}{l}\text { estándar de OTC sin } \\
\text { exposición a la luz }\end{array}$ & 12 & 7 & $0.012 \pm 0.013$ & $117.44 \pm 77.26$ & 0.9193 & 0.5601 \\
\hline Sedimento y & $\begin{array}{l}\text { OTC en alimento sin } \\
\text { exposición a la luz }\end{array}$ & 12 & & $0.009 \pm 0.009$ & $138.22 \pm 79.88$ & 0.9810 & 0.4033 \\
\hline con & $\begin{array}{l}\text { estándar de OTC } \\
\text { expuesto a la luz }\end{array}$ & 12 & & $0.016 \pm 0.008$ & $49.68 \pm 15.00$ & 0.9963 & 0.1693 \\
\hline & $\begin{array}{l}\text { OTC en alimento sin } \\
\text { exposición a la luz }\end{array}$ & 12 & & $0.012 \pm 0.004$ & $60.11 \pm 16.29$ & 0.9950 & 0.2043 \\
\hline
\end{tabular}

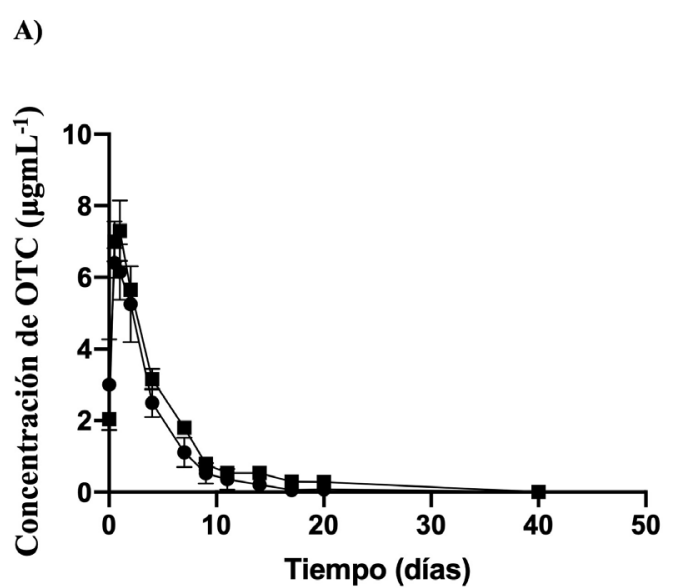

- Sedimento y agua marina expuestos a la luz

- Sedimento y agua marina sin exposición a la luz
B)

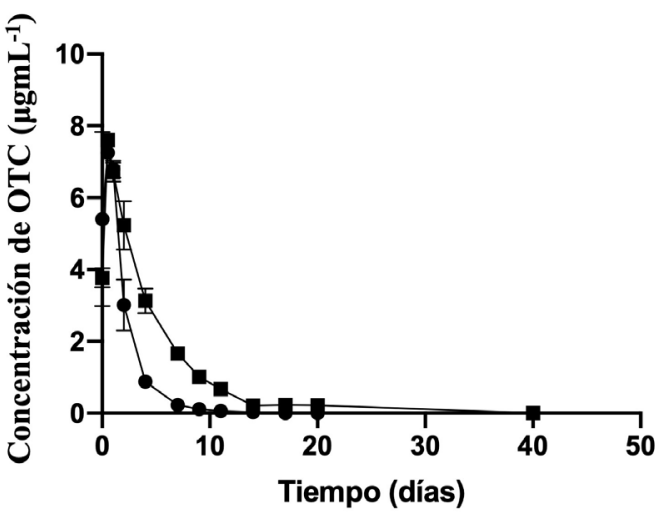

- Sedimento y agua marina expuestos a la luz

- Sedimento y agua marina sin exposición a la luz

Figura 3. Cinética de degradación de la OTC en alimento para camarón por efecto de la luz y pH en muestras de sedimento y agua marina. A) Degradación de la OTC a pH 7. B) Degradación de la OTC a pH 8.

El tiempo de vida media de la degradación del antibiótico en las muestras de sedimento y agua marina con alimento a pH 7 y exposición a la luz ocurrió a los $38.58 \pm 12.06$ min, mientras que en los frascos que no se expusieron a la luz se presentó a los $113.22 \pm 79.88 \mathrm{~min}$, indicando una degradación acelerada del antibiótico por fotolisis. El t $1 / 2$ de la OTC en los frascos con pH 8 y exposición a la luz se observó a los
$32.89 \pm 6.68 \mathrm{~min}$ y en los no expuestos, a los $60.11 \pm 16.29 \mathrm{~min}$ (Tabla III).

\section{Discusión}

Con base en los resultados obtenidos de la validación del método de análisis de OTC para asegurar la calidad de los datos generados, se demuestra la capacidad del método de 
cromatografía de líquidos de alta resolución, para generar resultados directamente proporcionales a la concentración del analito de interés, como lo establece Andrade (2018), ya que valores de $\mathrm{r}^{2}$ iguales o cercanos a la unidad demuestran la linealidad del método, así como la especificidad o selectividad del método analítico al no identificarse interferencia de impurezas presentes en la muestra, de acuerdo a lo observado en los cromatogramas. En el parámetro de exactitud, el porcentaje de recuperación obtenido, en las muestras con el antibiótico incorporado al sedimento y agua marina, fue comparable con los valores establecidos en los criterios de aceptación para métodos cromatográficos, que son de $80-110 \%$ de recuperación, de acuerdo a la guía ICH 2Q (R1) (ICH Harmonized Tripartite Guideline, 2005). En la cuantificación de la OTC el tiempo de retención cromatográfica del antibiótico fue similar al reportado por Pro (2016), quien obtuvo un tiempo de retención de $7 \mathrm{~min}$, utilizando cromatografía líquida de alta resolución.

La fotolisis e hidrólisis, son procesos que participan en la degradación de los antibióticos y son dependientes de la temperatura, el pH y la luz, como lo reportó Andrade (2018) y comprobado en este estudio a través del efecto de factores como la luz y la forma en que se encontraba la OTC utilizada (como estándar o incorporada en el alimento para camarón), influyendo directamente en la degradación del antibiótico $(P \leq 0.05)$. Sosa et al. (2013), reportaron que la OTC se distribuye en el agua durante las primeras $24 \mathrm{~h}$, como también se observó en nuestro estudio, alcanzando el antibiótico las más altas concentraciones entre las 12 y $24 \mathrm{~h}$ del inicio del ensayo. Factores como una alta salinidad y temperatura presentes en los estanques de cultivo, contribuyen a potenciar la lixiviación del antibiótico. La humedad elevada en el sedimento es un factor importante en la degradación de la OTC debido a que se metaboliza a sus epímeros de forma reversible en el carbono 4, dando origen a compuestos como epi-oxitetraciclina, anhidro-oxitetraciclina y epianhidro-oxitetraciclina, como ha sido reportado por Oka et al. (1989) y Li et al. (2019), quienes señalan que en un medio acuoso, la OTC es inestable y forma epímeros por hidrólisis.

La fotolisis es uno de los principales procesos de degradación de los antibióticos, en el caso de la OTC expuesta a la luz y a un $\mathrm{pH} 8$, la degradación fue más rápida y también por tener menor estabilidad en condiciones alcalinas, lo que coincide con lo reportado por Oka et al. (1989) y Halling-Sørensen, Sengeløv \& Tjørnelund (2002).

La presencia de residuos de antibióticos en los sistemas acuícolas puede incrementarse si estos fármacos son persistentes y se acumulan por adsorción en los lodos, sedimentos o suelos (Acevedo et al., 2015). La OTC se ha detectado en sedimentos acuícolas después de 3-6 meses de su administración a través del alimento (Samuelsen, 1989), y es reconocida por ser persistente en sedimentos acuícolas e inducir resistencia en la microflora y bacterias patógenas para los organismos acuáticos (Redrován, 2017). La residualidad de la OTC toma relevancia debido a que su concentración en las muestras de sedimento y agua marina se encontró por arriba del límite máximo permitido (LMP) para este antibiótico, de acuerdo a los valores de referencia, que son de $0.1 \mu \mathrm{gmL}^{-1}$ en la Unión Europea y $0.2 \mu \mathrm{gmL}^{-1}$ en Estados Unidos de América (Nogueira-Lima, Gesteira \& Mafezoli, 2006; FDA, 2014).

Estudios relacionados con la evaluación de la cinética de degradación de la OTC han demostrado que se incrementa a pH neutro, asociándolo a la formación de aniones de OTC capaces de absorber energía (Jiao et al., 2008). Sin embargo, autores como Liu et al. (2020), han mencionado que para demostrar el efecto del pH sobre la degradación de la OTC, la evaluación de este parámetro debe incluir mediciones de $\mathrm{pH}$ en rangos de 3 hasta 12, considerando que el antibiótico se degrada dependiendo de este factor. En este sentido, los datos de la degradación en nuestro estudio y la influencia del pH son justificados, ya que no mostraron diferencia significativa en el ANOVA multifactorial.

Por otra parte, Leal et al. (2019) mencionan que además de los factores como la salinidad presente en el agua de mar, la presencia de iones como $\mathrm{Mg}^{2+} \mathrm{o} \mathrm{Ca}^{2+}$, incrementan la cinética de degradación de la OTC, interactuando con las moléculas del fármaco y el efecto de la luz solar. Nuestros resultados mostraron una degradación más lenta del antibiótico, comparado con los hallazgos publicados por Leal et al. (2016), quienes estudiaron la fotodegradación de un estándar de OTC $\left(4 \mu \mathrm{gmL}^{-1}\right)$ en agua de mar proveniente de una granja acuícola y al aplicar una irradiación simulando la luz solar $\left(55 \mathrm{~W} / \mathrm{m}^{2}\right)$, se degradó el $80 \%$ del antibiótico en $21 \mathrm{~min}$, debido a la intensidad de la luz a la que fue expuesto. Aunado a esto, autores como Samuelsen, Lunestad, Ervik \& Fjelde (1994), han demostrado que la degradación de la OTC en sedimentos sin actividad antropogénica es baja, y su vida media está en función de la columna de agua. Es por ello, que algunos autores han recomendado que en sedimento y agua marina, el monitoreo de compuestos residuales y el mantenimiento de la actividad biológica son la clave para garantizar el funcionamiento de los ecosistemas sin un impacto ambiental considerable (Seo, Keum \& Li, 2009; Liu, Pan, Chen, Jiao \& Wang, 2012).

Los efectos más conocidos de la OTC en el medio acuático son la toxicidad para los organismos, la inhibición del crecimiento de las plantas acuáticas, disminución de la calidad del agua a nivel de eutrofización, así como el desarrollo de bacterias resistentes a estos antibióticos (Van der Grinten, Pikkemaat, van den Brandhof, Stroomberg \& Kraak, 2010; Acevedo et al., 2015). Lo anterior incrementa la preocupación por la incidencia que pueda tener este fármaco en los sistemas acuáticos. Por ello, es importante entender los procesos de 
degradación de la OTC y conocer su residualidad en los sistemas de cultivo, por ser éste principalmente utilizado en la industria acuícola.

Es difícil predecir el impacto que tienen los antibióticos en los ecosistemas, sin embargo, su presencia puede provocar alteraciones en la cadena trófica con una posible bioacumulación a largo plazo, selección de bacterias patógenas resistentes y genotoxicidad, entre otros posibles efectos, razones por las cuales su presencia es considerada un problema ambiental emergente a nivel mundial (Espinosa \& Bermúdez, 2012). Es importante mencionar que los antibióticos que son administrados en los sistemas de cultivo acuícola, no eliminan la totalidad de las bacterias, la cantidad de bacterias disminuye, pero un porcentaje de ellas puede sobrevivir al efecto del antibiótico, lo que se conoce como persistencia bacteriana (Becerra, Plascencia, Luévanos, Domínguez \& Hernández, 2009), esto puede ocurrir en bacterias patógenas presentes en ambientes acuícolas, y causar serios problemas en esta industria y en el medio ambiente, ya que la degradación del antibiótico puede no ser completa. Por ello, es recomendable la remoción del sedimento en los estanques de cultivo una vez concluido el ciclo de producción, con el propósito de asegurar la exposición del fármaco a la luz y con ello favorecer la degradación por fotolisis en el medio ambiente. Cabe resaltar que es poca la información publicada acerca del comportamiento y residualidad de la OTC bajo condiciones reales de cultivo de organismos marinos, donde se pueden tener distintas condiciones de $\mathrm{pH}$ y salinidad, y aún menores son los estudios acerca del comportamiento de la OTC en alimento para camarón, por lo que esta investigación es importante debido al conocimiento que genera acerca de la fotodegradación del antibiótico, en un estudio de laboratorio al simular las condiciones de los estanques de las granjas acuícolas.

\section{Conclusiones}

Se establecieron las condiciones cromatográficas óptimas para la detección y cuantificación de la oxitetraciclina en muestras de sedimento y agua marina mediante cromatografía de líquidos de alta resolución, obteniéndose valores adecuados en los parámetros de validación, lo que asegura la confiabilidad de los resultados obtenidos en la cuantificación de la OTC. Es importante resaltar que el método es sensible, y tiene la capacidad de detectar concentraciones de OTC en el nivel del límite máximo permitido de acuerdo a las regulaciones, por lo que resulta una herramienta útil para llevar a cabo el monitoreo de este antibiótico en estanques de cultivo de camarón.

Los resultados del tratamiento de adición de OTC como estándar sin exposición a la luz, mostraron una degradación del antibiótico de $88.33 \%$ y $90.90 \%$ a condiciones de $\mathrm{pH}$ de 7 y 8 respectivamente, a los 40 días del ensayo. Mientras que en el tratamiento con el estándary exposición a la luz, la degradación fue del $100 \%$ para ambos valores de $\mathrm{pH}$.
En el tratamiento de adición de la OTC en el alimento para camarón con y sin exposición a la luz bajo condiciones de pH 7 y 8 , la degradación fue del $100 \%$, a los 40 días. Este hallazgo es favorable, ya que en las granjas de cultivo de camarón, la administración de los antibióticos se lleva a cabo a través del alimento.

El tiempo de vida media de degradación de la OTC en el alimento, fue menor con respecto a las muestras adicionadas con estándar con y sin exposición a la luz.

El uso de antibióticos en la acuicultura representa una vía de entrada de contaminantes a los ambientes acuáticos y terrestres, los sedimentos actúan como una fuente potencial de acumulación de diversos compuestos químicos y el agua tiene un importante papel en la transformación y transporte de los contaminantes, incluyendo residuos de antibióticos, bacterias resistentes y acelerar la evolución y diseminación de genes de resistencia a los antibióticos, lo que puede ocasionar un impacto negativo al ambiente, aumentando el riesgo de transmisión a humanos y animales.

La presencia de residuos de antibióticos en los sedimentos es relevante, ya que muchos de estos compuestos han demostrado ser persistentes en el suelo, el sedimento y el agua, lo que representa riesgos ecológicos a largo plazo, y aunque se encuentren en concentraciones traza, la exposición crónica sobre la microbiota acuática puede provocar la generación de resistencia bacteriana.

\section{REFERENCIAS}

Acevedo, R. L., Severiche, C.A. \& Jaimes, J. C. (2015). Bacterias resistentes a antibióticos en medios acuáticos. Producción Limpia, 10(2), 160-172. http://www.scielo.org.co/pdf/pml/ v10n2/v10n2a15.pdf.

Andrade, C. A. (2018). Remoción de oxitetraciclina presente en soluciones acuosas usando cenizas de cáscara de arroz (Tesis de maestría). Instituto politécnico de Leiria, pág. 8-11, 108-118. Ecuador. https://core.ac.uk/download/ pdf/161394998.pdf.

Andreozzi, R., Raffaele, M. \& Nicklas, P. (2003). Pharmaceutical in STP effluents and their solar photodegradation in aquatic environment. Chemosphere, 50, 1319-1330. https://doi. org/10.1016/S0045-6535(02)00769-5.

Becerra, G., Plascencia, A., Luévanos, A., Domínguez, M. \& Hernández, I. (2009). Mecanismo de resistencia a antimicrobianos en bacterias. En: Enfermedades Infecciosas y Microbiología. 29(2),70-76. https://www.medigraphic. com/pdfs/micro/ei-2009/ei092e.pdf.

Doi, A. \& Stoskopf, M. (2000). The Kinetics of oxytetracycline degradation in deionized water under varying temperature, $\mathrm{pH}$, light, substrate, and organic matter. Journal of Aquatic Animal Health, 12, 246-253. https://doi.org/10.1577/15488667(2000)012<0246:TKOODI $>2.0 . C O ; 2$. 
Espinosa, P. A. \& Bermúdez, A. M. C. 2012. La acuicultura y su impacto al medio ambiente. Estudios Sociales, 20(2), 221232. https://www.redalyc.org/articulo.oa?id=41724972010.

Food and Drug Administration (FAO) (2018). The state of world fisheries and aquaculture 2018-Meeting the sustainable development goals. http://www.fao.org/3/i9540en/i9540en. pdf.

FDA (2011). Tolerance for residues of new animal drugs in food. Electronic Code of Federal Regulation. Food and Drugs. Recuperado de: https://www.fda.gov/media/80297/ download

FDA. (2014). Tolerance for residues of new animal drugs in food. Electronic Code of Federal Regulation. Food and Drugs. Recuperado de: http://www.ecfr.gov/cgi.

Gómez-Gil, B., Roque,A. \& Guerra,A. L. (2014). Enfermedades infecciosas más comunes en México y el impacto del uso de antimicrobianos. En Páez O. F. (Ed.) Camaronicultura y Medio Ambiente. (pp. 315-346). Universidad Nacional Autónoma de México. http://cesaibc.org/pdf/infointeres/ crustaceos/enfermedadesmexico.pdf.

González-Gaya, B., Cherta, L., Nozal, L. \& Rico, A. (2018). An optimized simple treatment method for the determination of antibiotics in seawater, marine sediments and biological samples using LC-TOF/MS. Science Total Environment, 1(643), 994-1004. https://doi.org/10.1016/j. scitotenv.2018.06.079.

Halling-Sørensen, B., Sengeløv, G. \& Tjørnelund, J. (2002). Toxicity of tetracyclines and tetracycline degradation products to environmentally relevant bacteria, including selected tetracycline-resistant bacteria. Archives of Environmental Contamination and Toxicology.42(3), 263271. https://doi.org/10.1007/s00244-001-0017-2.

Hong-Thih, L. \& Jing-Ju, L. (2009). Degradation of oxolinic acid and flumequine in aquaculture pond waters and sediments. Chemosphere, 75(4), 462-468. https://doi.org/10.1016/j. chemosphere.2008.12.060.

Institute T. N. V. (2016). Use of antibiotics in Norwegian aquaculture on behalf of Norwegian seafood council. The Norwegian Veterinary Institute. Oslo.

Harmonized Tripartite Guideline (ICH). (2005). Validation of analytical procedures: text and methology. ICH 2QR1. https://www.ema.europa.eu/en/ich-q2-r1-validationanalytical-procedures-text-methodology.

Jiao, S. J., Meng, D. Q., Yin, D. Q., Wang, L. H. \& Chen, L. Y. (2008). Aqueous oxytetracycline degradation and the toxicity change of degradation compounds in photoirradiation process. Journal of Environmental Sciences. 20(7), 806-820. https://doi.org/10.1016/S10010742(08)62130-0.

Kong, W., Li, C., Dolhi, J. M., Li, S., He, J. \& Qiao, M. (2012). Characteristics of oxytetracycline sorption and potential bioavailability in soils with various physical-chemical properties. Chemosphere, 87(5), 542-548. https://doi. org/10.1016/j.chemosphere.2011.12.062.
Kulshrestha, P., Giese, R. F. \& Aga, S. R. (2004). Investigating the molecular interactions of oxytetracycline in clay and organic matter: insights on factors affecting its mobility in soil. Environmental Science of Technology, 38(15), 40974105. https://doi.org/10.1021/es034856q.

Lai, H. \& Lin, J. (2009). Degradation of oxolinic acid and flumequine in aquaculture pond waters and sediments. Chemosphere, 75(4), 462-468. https://doi.org/10.1016/j. chemosphere.2008.12.060.

Leal, J. F., Esteves, V. I. \& Santos, E. B. (2016). Use of sunlight to degrade oxytetracycline in marine aquaculture's waters. Environmental Pollution, 213, 932-939. https://doi. org/10.1016/j.envpol.2016.03.040.

Leal, J. F., Esteves, V. I. \& Santos, E. B. H. (2019). Solar photodegradation of oxytetracycline in brackish aquaculture water: New insights about effects of $\mathrm{Ca}^{+}$ and $\mathrm{Mg} 2^{+}$. Journal of photochemistry and photobiology A: Chemistry, 372(3), 218-225. https://doi.org/10.1016/j. jphotochem.2018.12.022.

Li, Z., Qi, W., Feng, Y., Liu, Y., Ebrahim, S. \& Long, J. (2019). Degradation mechanisms of oxytetracycline in the environment. Journal of Integrative Agriculture, 18(9), 1953-1960. https://doi.org/10.1016/S20953119(18)62121-5.

Li, E., Chen, C., Zeng, N., Yu, Z., Xiong, X. \& Chen-Quin, J. G. (2008). Comparison of digestive and antioxidant enzymes activities, haemolymph oxyhemocianin contents and hepatopancreas histology of White shrimp, Litopenaeus vannamei at various salinities. Aquaculture, 274(1), 80-86. https://doi.org/10.1016/j.aquaculture.2007.11.001.

Li, J. \& Zhang, H. (2016). Adsorption-desorption of oxytetracycline on marine sediments: Kinetics and influencing factors. Chemosphere, 164, 156-163. https:// doi.org/10.1016/j.chemosphere.2016.08.100.

Liu, W., Pan, N., Chen, W., Jiao, W. \& Wang, M. (2012). Effect of veterinary oxytetracycline on functional diversity of soil microbial community. Plant Soil Environent, 58(7), 295 301. https://doi.org/10.17221/430/2011-PSE.

Liu, X., Zhang, H., Luo, Y., Zhu, R., Wang, H. \& Huang, B. (2020). Sorption of oxytetracycline in particulate organic matter in soils and sediments: Roles of $\mathrm{pH}$, ionic strength and temperature. Science of the Total Environment, 714, 136628. https://doi.org/10.1016/j.scitotenv.2020.136628

Nogueira-Lima, A., Gesteira, T. \& Mafezoli, J. (2006). Oxytetracycline residues in cultivated marine shrimp (Litopenaeus vannamei Boone, 1931) (Crustacea, Decapoda) submitted to antibiotic treatment. Aquaculture, 254(1-4), 748-757. https://doi.org/10.1016/j. aquaculture.2005.11.021.

Oka, H., Ikai, Y., Kawamura, N., Yamada, M., Harada, K., Ito, S. \& Suzuki, M. (1989). Photodecomposition products of tetracyclines in aqueous solution. Journal of Agricultural and Food Chemistry, 37(1), 226-231. https:// doi.org/10.1021/jf00085a052. 
Oka, H., Matsumoto, H., Uno, K., Harada, K., Kadowaki, S. \& Suzuki, M. (1985). Improvement of chemical analysis of antibiotics VIII. Application of prepacked C18 cartridge for the analysis of tetracycline residues in animal liver. Journal of Chromatography A, 325(1), 265-274. https:// doi.org/10.1016/S0021-9673(00)96027-8.

Pouliquen, H., Delépée, R., Larhantec-Verdier, M., Morvan, M. \& Le, H. (2007). Comparative hydrolysis and photolysis of four antibacterial agents (oxytetracycline, oxolinic acid, flumequine and florfenicol) in deionized water, freshwater and seawater under abiotic conditions. Aquaculture, 262(1), 23-28. https://doi.org/10.1016/j.aquaculture.2006.10.014.

Pouliquen, H., LeBris, H. \& Pinault, L. (1992). Experimental study of the therapeutic application of oxytetracycline, its attenuation in sediment and sea water and implications for farm culture of benthic organisms. Marine Ecology Progress Series, 89(1), 93-98. https://doi.org/10.3354/meps089093.

Prado, S., Romalde, J. L. \& Barja, J. L. (2010). Review of probiotics for use in bivalve hatcheries. Veterinary Microbiology, 145(3-4), 187-197.https://doi.org/10.1016/j. vetmic.2010.08.021.

Pro, F. J. (2016). Valoración de efectos ecotoxicológicos de oxitetraciclina en organismos terrestres y acuáticos mediante el empleo de sistemas multi-especie en suelo (MS3). (Tesis maestría). Universidad Complutense de Madrid, España. (pp.95-96). https://eprints.ucm.es/38767/.

Redrován, K. (2017). Medidas terapéuticas para el control de vibriosis en el cultivo de camarón blanco Litopenaeus vannamei (Tesis de maestría). Universidad Técnica de Machala, Ecuador. (pp.19-24). http://repositorio.utmachala. edu.ec/handle/48000/11355.

Rojas, D. (2010). Tolerancia a oxitetraciclina de comunidades microbianas de sedimentos en muestras provenientes de proyectos agropecuarios en el Distrito de Riego Arenal Tempisque. (Tesis de licenciatura). Universidad de Costa Rica. Costa Rica. (pp. 17-18). http://hdl.handle. net/10669/16837.

Samuelsen, O. B. (1989). Degradation of oxytetracycline in seawater at two different temperatures and light intensities, and the persistence of oxytetracycline in the sediment from a fish farm. Aquaculture, 83(1-2), 7-16. https://doi. org/10.1016/0044-8486(89)90056-2.

Samuelsen, O. B., Lunestad, B. T., Ervik, A. \& Fjelde, S. (1994). Stability of antimicrobial agents in artificial marine sediment studied under laboratory conditions. Aquaculture, 126(3-4), 283-290. https://doi.org/10.1016/0044-8486(94)90044-2.

Seo, S., Keum, Y. S. \& Li, Q. X. (2009). Bacterial degradation of aromatic compounds. International Journal of Environmental Research and Public Health, 6(1), 278-309. https://doi.org/10.3390/ijerph6010278.

Sosa, D., Medina, A. \& Faure, R. (2013). Empleo de la oxitetraciclina en el cultivo del camarón con énfasis en la especie Litopenaeus vannamei y alternativas que favorecen la disminución o sustitución de su aplicación. Revista Electrónica de Veterinaria, 14(7), 1-11. https://www. redalyc.org/pdf/636/63628041010.pdf.

Soto-Rodríguez, S. A., Gómez-Gil, B., Roque, A. \& Lozano, R. (2008). MIC'S de antibióticos de Vibrio spp aislados de L. vannamei cultivado en México. Panorama Acuícola Magazine. 14(1), 52-57. https:/www.researchgate.net/ publication/284980299 MICs de antibioticos de Vibrio spp aislados del L. vannamei cultivado en México.

Tirado, D., Montero, P. \& Acevedo, D. (2015). Estudio comparativo de métodos empleados para la determinación de humedad de varias matrices alimentarias. Información Tecnológica, 26(2), 3-10.

Topp, E., Joakim, L. D. G., Miller, D. N., Van den Eede, C. \& Virta, M. P. J. (2018). Antimicrobial resistance and the environment: assessment of advances, gaps and recommendations for agriculture, aquaculture and pharmaceutical manufacturing. FEMS Microbiology Ecology, 94(3), 1-5. https://doi.org/10.1093/femsec/ fix 185 .

Van der Grinten, E., Pikkemaat, M. G., van den Brandhof, E. J., Stroomberg, G. J. \& Kraak, M. H. (2010). Comparing the sensitivity of algal, cyanobacterial and bacterial bioassays to different groups of antibiotics. Chemosphere, 80(1), 1-6. https://doi.org/10.1016/j.chemosphere.2010.04.011.

Venkateswara-Rao, A. (2009). Vibriosis en la acuacultura del camarón. India. Recuperado de: https://aquahoy. com/archivo/156-uncategorised/7165-vibriosis-en-laacuicultura-del-camaron

Yan, W., Guo, Y., Xiao, Y., Wang, S., Ding, R., Jiang, J., Gang, H., Wang, H., Yang, J. \& Zhao, F. (2018). The changes of bacterial communities and antibiotic resistance genes in microbial fuel cell during long-term oxytetracycline processing. Water Research, 142, 105-114. https://doi. org/10.1016/j.watres.2018.05.047. 